\title{
Lower multisensory temporal acuity in individuals with high schizotypal traits:
}

\section{a web-based study}

\author{
Gianluca Marsicano $^{1,2, \pi}$, Filippo Cerpelloni ${ }^{3,4,5}$ II David Melcher $^{3,6, *}$, and Luca
}

\section{Ronconi $^{1,2}$}

${ }^{1}$ School of Psychology, Vita-Salute San Raffaele University, Milan, Italy.

${ }^{2}$ Division of Neuroscience, IRCCS San Raffaele Scientific Institute, Milan, Italy.

${ }^{3}$ Center for Mind/Brain Sciences and Department of Psychology and Cognitive Science, University of Trento, Rovereto, Italy.

${ }^{4}$ Laboratory of Biological Psychology, Department of Brain and Cognition, Leuven Brain Institute, KU Leuven, Leuven, Belgium

${ }^{5}$ Institute of research in Psychology (IPSY) \& Institute of Neuroscience (IoNS) - University of Louvain (UCLouvain), Louvain, Belgium

${ }^{6}$ Psychology Program, Division of Science, New York University Abu Dhabi, United Arab Emirates.

II = equally contributing authors/ co-first authorship

*Corresponding author:

David Melcher (dm93@nyu.edu), Psychology Program, Division of Science, New York University Abu Dhabi, United Arab Emirates 


\section{ABSTRACT}

Natural events are often multisensory, requiring the brain to combine information from the same spatial location and timing, across different senses. The importance of temporal coincidence has led to the introduction of the temporal binding window (TBW) construct, defined as the time range within which multisensory inputs are highly likely to be perceptually bound into a single entity. Anomalies in TBWs have been linked to confused perceptual experiences and inaccurate filtering of sensory inputs coming from different environmental sources. Indeed, larger TBWs have been associated with disorders such as schizophrenia and autism and are also correlated to a higher level of subclinical traits of these conditions in the general population. Here, we tested the feasibility of using a webbased version of a classic audio-visual simultaneity judgment (SJ) task with simple flash-beep stimuli in order to measure multisensory temporal acuity and its relationship with schizotypal traits as measured in the general population. Results show that: (i) the response distribution obtained in the web-based SJ task was strongly similar to those reported by studies carried out in controlled laboratory settings, and (ii) lower multisensory temporal acuity was associated with higher schizotypal traits in the "disorganized" domains. Our findings reveal the possibility of adequately using a web-based audio-visual SJ task outside a controlled laboratory setting, available to a more diverse and representative pool of participants. These results provide additional evidence for a close relationship between lower multisensory acuity and the expression schizotypal traits in the general population. 


\section{INTRODUCTION}

In daily life our brains constantly encode, filter and integrate information from different sensory modalities, in such a way as to give order to our perceptual experience. This process, called multisensory integration, is an essential feature of human cognition which allows the input in the environment from different sensory modalities to be correctly integrated into a coherent, unitary percept, while also correctly segmenting sensory information from different events as separate and unique events (Stevenson et al., 2014, Murray et al. 2016; Pasqualotto, Dumitru \& Myachykov, 2016). Multisensory perception, then, forms an important foundation in constructing our perceptual experience in terms of specific objects, events and social interactions.

A key aspect is to correctly perceive information coming from different sensory modalities about the same object/event as belonging to a single event, rather than as separate events (Spence, 2007), facilitating the skills of detection (Lovelace et al., 2003, Diederich \& Colonius, 2015) and localization (Wilkinson et al. 1996), in order to adapt behavior to environmental demands (Diederich and Colonius 2004). Multisensory integration is not limited only to the processing of simple sensory stimuli, but yields beneficial cascade effects also in higher-order cognitive and social processes, which translate into an improvement of the functions such as speech perception (Bishop \& Miller, 2009; Grant \& Seitz, 2000) and complex verbal and non-verbal problem solving, reasoning and thoughts (Zmigrod \& Zmigrod, 2016).

This integrative ability is strongly dependent on the spatiotemporal relationship and on the physical characteristics of stimuli of different sensory origin (Wallace et al. 2004, Matthews, Stewart, \& Wearden, 2011), factors that act as statistical indices of the possibility that the stimuli are paired together. Such regularities increase the likelihood that stimulation to different senses actually comes from the same spatiotemporal event.

A greater spatial and temporal proximity of the stimuli facilitates multisensory interactions (Stevenson et al., 2013). The importance of these factors is evident at various levels of investigation, as shown by numerous neuroimaging (Royal et al., 2009; Senkowski et al. 2007; Schall et al., 2009; 
Talsma et al. 2009; Stevenson et al., 2011) and psychophysical (Zampini et al. 2005; Conrey and Pisoni 2006; van Atteveldt et al. 2007; van Wassenhove et al. 2007; Powers et al. 2009; Foss-Feig et al. 2010; Hillock et al. 2011; Stevenson et al. 2012; Hillock-Dunn \& Wallace, 2012; Kuling et al., 2012) studies. Critically, the temporal distance between stimuli of different sensory modalities determines the occurrence of the multisensory integration process, reflecting the key role of temporal processing for accurate multisensory integration (Zampini et al., 2005; Stevenson et al., 2012). Critically, multisensory integration not only occurs when the two stimuli are presented perfectly synchronously, but also when they are not exactly synchronous, so that the multisensory input can be integrated and perceived as a unitary percept (Andersen et al. 2004; Vatakis \& Spence 2007). This is the case for audiovisual stimuli, where such temporal "tolerance" allows them to be integrated into a single integrated percept, providing order for the perceptual experience, and responsible for accurate filtering of the auditory and visual input coming from different events (Wallace and Stevenson, 2014). Perceptual binding of audiovisual stimuli is likely to occur only if they are presented in proximity within a limited temporal interval, a process that is operationalized in the construct of the "temporal binding window" (TBW) (Colonius and Diederich 2004; Hairston et al. 2005; Stevenson et al. 2012, Wallace et al., 2014; Noel et al., 2016). The width of this temporal window is commonly used as a proxy measure for multisensory temporal acuity. The TBW is a probabilistic construct, defined by the range of stimulus onset asynchronies (SOAs) in which stimuli of different sensory modalities are likely to be perceptually paired.

Interestingly, perceiving two stimuli as simultaneous does not require that they are presented at the same time and perceived simultaneity can even be greater when stimuli from one modality arrive prior to another. Experimental manipulation of the "leading" sensory modality, that is the order in which the audiovisual stimuli are presented, influences the judgment of simultaneity. At the behavioral level, these asymmetries in the distribution obtained from the simultaneity rate indicate a narrower size of the binding window for Auditory-Leading (AL) as compared to Visual-Leading (VL) trials (Cecere, Gross, \& Thut, 2016). At the neural level, these asymmetries are characterized by two 
distinct patterns of brain activity, which reveal that potentially different mechanisms support different multisensory processes (Cecere et al., 2017). Furthermore, the spatial position of the presentation of stimuli influences the perception of simultaneity, in a way that auditory and visual stimuli are more likely to be integrated into a single percept and judge as simultaneous, when presented in congruent spatial positions, rather than in different locations (Zampini et al., 2005).

Using similar paradigms, recent studies have revealed that this temporal window in multisensory processing within the general population is not homogeneous, but is characterized by a high interindividual variability (Ferri et al., 2017). Where a narrower TBW reflects higher accuracy in multisensory integration (Martin et al., 2013; Zmigrod and Zmigrod, 2016), a wider TBW indicates lower multisensory acuity/resolution, which may result in an ambiguous perceptual experience and sensory overload (Sartorato et al., 2017; Ferri et al., 2017; Ferri et al., 2018), in a confused sense of self (Postmes et al., 2014) or even to hallucinations (Stevenson et al., 2017).

This reduced precision in the multisensory integration process is typical in the clinical population affected by Schizophrenia (SCZ), where an enlarged TBW is associated with socio-cognitive and perceptual differences (Zhou et al., 2018), resulting in an aberrant synchrony perception of audiovisual stimuli that likely determines an ambiguous and imprecise perceptual experience (Tseng et al., 2015; Balz et al., 2016; Stevenson \& Wallace; 2018, Haß et al., 2017). Moreover, sub-clinical traits of SCZ are also present in the non-clinical population (Ettinger et al., 2014), with higher traits reflecting a higher prevalence and intensity of behavioural characteristics associated with SCZ. Interestingly, the deficits in the temporal processes of multisensory integration have recently been identified also in this non-clinical population with higher schizotypal traits (Ferri et al., 2018; Fenner et al., 2020). For example, one recent study has shown that a lower temporal acuity in multisensory integration is correlated to a higher level of schizotypal symptomatology, and that this relationship is specific for the cognitive-perceptual domain (Dalal, Muller \& Stevenson, 2021). These studies suggest the need to extend the investigation of multisensory temporal processing anomalies also to the general population with a different degree of schizotypal traits. 
In line with this evidence, in the current study, we have employed an online version of the Simultaneity Judgment (SJ) task, which is one of the main experimental paradigms developed to investigate TBW, in which participants are asked to judge the perceived simultaneity of visual and auditory stimuli presented in congruent or incongruent spatially position across different SOAs (Zampini et al., 2005; Vatakis et al. 2008; Roach et al. 2011). The rate of perceived simultaneity responses across different SOAs allows estimating the width of the TBW. Hence, the individual's TBW indicates the time range in which subjects show a high probability of judging two stimuli as simultaneous, and is typically defined as a function of the time interval between the $75 \%$ thresholds observed AL and VL trials (Stevenson et al., 2017).

First of all, our aim is to demonstrate the possibility of conducting a typical lab-based psychophysical task through a web-based platform, in a less controlled setting. The need and the possibility of adapting lab-based experiments to online context derives from the advantages offered by this system. Indeed, it is possible to make research more accessible to a greater number of participants, to increase their diversity within the recruited sample (i.e. age, gender, origin, culture and social status) and to optimize resource efficiency and timing of administration (Mason \& Suri, 2011; Berinsky et al., 2012; Buhrmester et al., 2016). Importantly, the use of this system allows recruiting larger samples that does not require a higher workload (Reips, 2002; Cohen et al., 2007; Sauter, Draschkow \& Mack, 2020). In line with previous lab-based evidence in the literature (e.g. Zampini et al, 2005; Vatakis et al. 2008), we expect that the multisensory integration process of audiovisual stimuli will vary according to the SOAs. Increasing the temporal synchrony of audiovisual stimuli would lead to a high degree of perceived simultaneity of audiovisual stimuli and multisensory integration compared to trials in which the SOA is larger. We also expect a generally greater multisensory temporal acuity when stimuli are presented in the same spatial position, rather than in different locations. Based on recent evidence (Cecere, Gross, \& Thut, 2016; Cecere et al., 2017), we will also focus our attention on the possible asymmetries in the multisensory integration processes deriving from the leading sense (AL vs VL). 
Alongside the behavioral web-based task, participants were asked to complete the Schizotypal Personality Questionnaire (SPQ; Raine 1991; Fossati et al. 2003), to analyze the relationship between schizotypal traits in the general population and multisensory temporal acuity. Based on recent evidence (Ladal et al., 2021), we hypothesized that a lower multisensory temporal acuity - i.e. an enlarged TBW - would correlate with higher levels of schizotypal traits.

It is worth noting that some theories of schizophrenia suggest that development of clinical levels of SCZ is linked to both genetics and to environmental stressors, with less stressors potentially leading to schizotypy rather than full schizophrenia (for example: Mirsky and Duncan, 1986; van Os, Kenis \& Rutten, 2010). If so, then widespread screening of schizotypal traits using a simple web-based task might be useful for identifying markers for at-risk individuals.

\section{METHODS}

\section{Participants}

A total of 55 participants took part initially in the study. All were volunteers and presented normal or corrected to normal vision and hearing. Four subjects were excluded from analyses due to inability to do the task, as they did not reach a 50\% mean rate of simultaneity in at least one of the conditions. The final sample of participants included in the analyses comprised 51 participants $(26 \mathrm{~F}$, mean age $=$ 23.9, $\mathrm{SD}=3.26$ ). All participants were provided with a document containing details about the procedure for completing the questionnaire and the web-based version of the audiovisual SJ task. In particular, we underlined the importance of following the instructions we provided for an optimal execution of the online task, where we stressed the importance of sitting in a quiet room, using headphones/earphones at a comfortable volume. We specified that the link could be used only from a PC and not from mobile devices. The research project was approved by the Ethical Committee of the University of Trento, and all participants gave their informed consent.

\section{Questionnaire for schizotypal traits}


Alongside the behavioral experiment participants were asked to complete the Schizotypal Personality Questionnaire (SPQ; Raine 1991; Fossati et al. 2003) - presented online through the Google Forms platform. This self-report questionnaire aimed at estimating the presence of schizotypal traits and is composed of 74 questions in which participants are asked to answer questions regarding different aspects of their personality, in addition to questions concerning sensorial experiences and beliefs, with "Yes" or "No" statements. In particular, the test contains nine subscales, divided into three components: Cognitive-Perceptual (subscales: ideas of reference, unusual perceptual experiences, magical thinking, suspiciousness), Disorganization (subscales: odd speech, odd or eccentric behavior) and Interpersonal (subscales: excessive social anxiety, no close friends, constricted affect, suspiciousness).

\section{Online audiovisual Simultaneity Judgement (SJ) task}

The task was administered using Pavlovia.org in combination with PsychoPy (Peirce, 2007, 2008), a web-based platform for the presentation of psychophysics experiments via common web browsers. This allowed us to collect remotely data for the SJ task. The audiovisual stimulus pairs were initially created in the form of videos using Matlab 2020a and then manipulated with Blender (https://www.blender.org/) in order to create the different SOA conditions. This strategy was chosen after pilot testing because it was the most reliable across web browsers and operating systems.

To investigate spatial position effects, visual stimuli were presented alternatively on the left or right side of the screen and auditory stimuli on either the left or right headphones/earbuds channel. To explore the extent of the TBW, a fixed array of SOAs was used $( \pm 350, \pm 300, \pm 250, \pm 200, \pm 150, \pm 100$, $\pm 50,0 \mathrm{~ms}$ ) presenting either the auditory (AL trials) or the visual (VL trials) stimulus first (Fig. 1). The two order conditions were balanced across trials. Assuming that participants kept the recommended distance of $50 \mathrm{~cm}$ from the screen, the visual stimulus employed was a gaussian blob with a diameter of 5 degree of visual angle, presented at approximately 18 deg of eccentricity from the central fixation point. All visual stimuli lasted for a total of $34 \mathrm{~ms}$, equivalent to 2 frames at 60 
$\mathrm{Hz}$ (the refresh rate of common PCs/laptops). As part of the data collection process, the refresh rate of the monitor/display was recorded for each participant. After data collection, we confirmed that all participants performed the task at this refresh rate with no exceptions. The auditory stimulus was a pure tone at $750 \mathrm{~Hz}$ with a duration of $34 \mathrm{~ms}$, to match the visual counterpart.

In each trial a fixation point was presented at the center of the screen for a fixed time of $1300 \mathrm{~ms}$ and a variable time between 150 and $500 \mathrm{~ms}$ before the appearance of the first stimulus. After each trial, participants were asked to rate the simultaneity of auditory and visual stimuli on a 5-point scale, with rating 1-2 indicating the presence/absence of simultaneity $(1=$ certainly synchronous; $2=$ probably synchronous), ratings 4-5 indicating simultaneity ( $4=$ probably asynchronous; 5 = certainly asynchronous), and rating 3 indicating a 'not sure/not seen' response. The latter was later discarded from analyses as non-informative.

[Insert Figure 1 about here] 


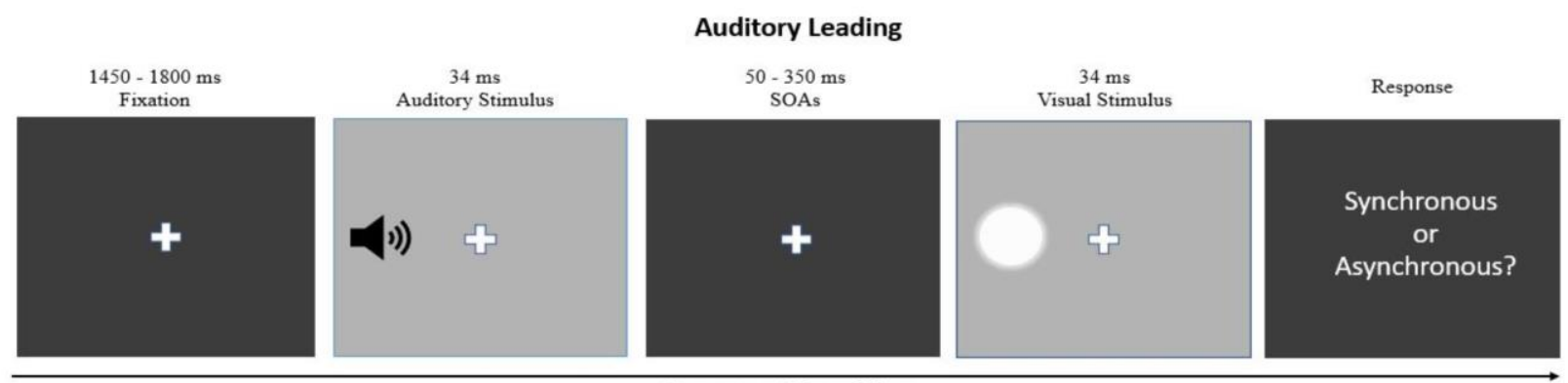

Congruent Condition

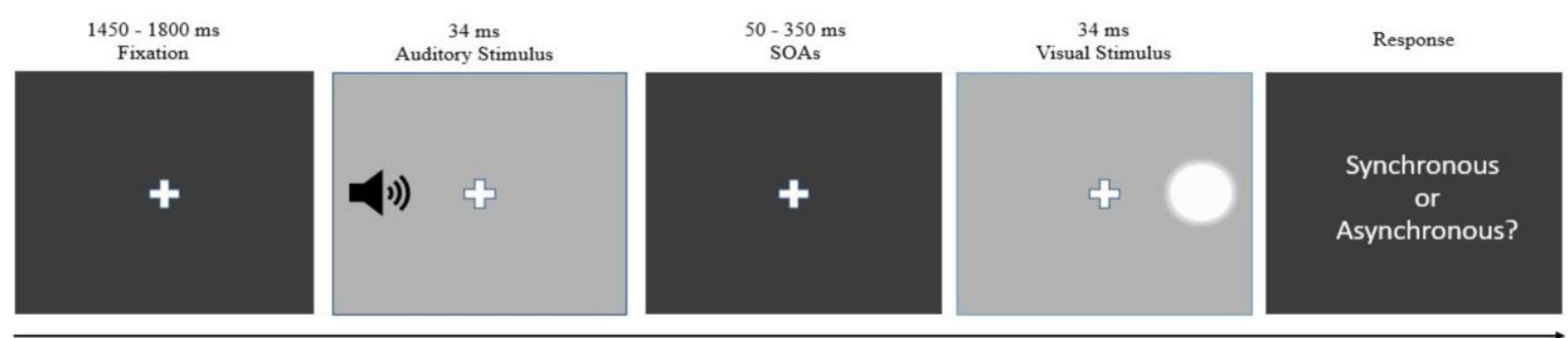

Incongruent Condition

\section{Visual Leading}
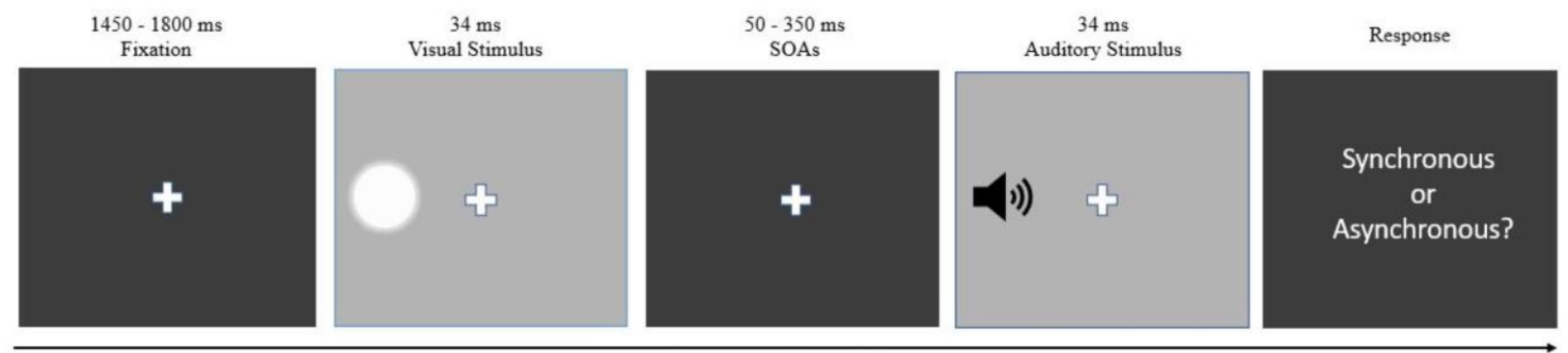

Congruent Condition
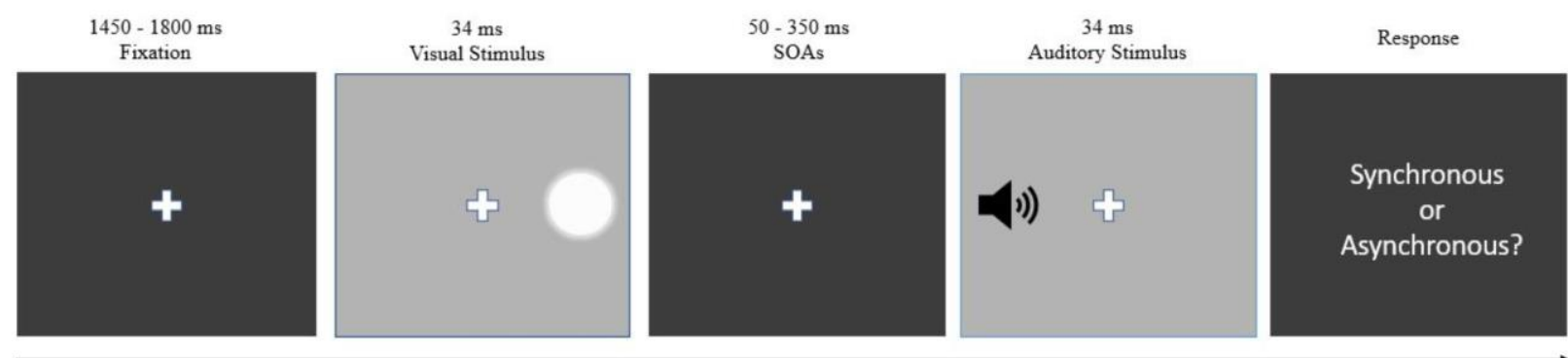

Incongruent Condition

Figure 1 Schematic representation of the web-based Simultaneity Judgment task. In each trial a fixation point is presented at the center of the screen for a variable time between 1450 and $1800 \mathrm{~ms}$ before the appearance of the first stimulus. A fixed array of SOAs between the first and second stimulus was used across trials $( \pm 350, \pm 300, \pm 250, \pm 200, \pm 150, \pm 100$, $\pm 50,0 \mathrm{~ms})$. Trials were counterbalanced between the following two conditions: A) Auditory Leading (AL) trials: when the auditory stimulus was followed by the visual stimulus, B) Visual Leading (VL trials): when the visual stimulus was followed by the auditory stimulus. The stimuli were presented alternatively on the left or right side of the screen and auditory stimuli on either the left or right headphones/earphones channel, leading to a spatially congruent or incongruent stimulus presentation.

\section{Data analysis}


We first performed a repeated measures Analysis of Variance (ANOVA) on the rate of synchronous responses with the aim of testing whether performance was influenced by the spatial congruency factor (two levels: congruent vs. incongruent) and SOAs (fifteen levels: $-350,-300,-250,-200,-150$, $-100,-50,0,+50,+100,+150,+200,+250,+300,+350)$, which were used as within-subjects factors. In a second step, we obtained the individual $75 \%$ thresholds values from the fitting of the psychometric logistic curve for each subject, separately for the AL and the VL condition, and for each spatial congruency condition (congruent vs. incongruent). Paired samples t-tests were used to investigate the differences in simultaneity rates (separately for AL and VL trials, with SOAs ranging from -350 to $-50 \mathrm{~ms}$ and from 50 to $350 \mathrm{~ms}$, respectively) and thresholds as a function spatial congruency. With the purpose of testing interindividual differences in multisensory temporal acuity and as a function of schizotypal traits, following previous studies we additionally calculated the width of the TBW as the sum for each participant of the absolute $75 \%$ threshold values for AL and VL trials.

Measures derived from the SJ task (i.e. simultaneity rate, thresholds/TBW obtained from the psychometric fitting) and the scores obtained in the SPQ (including the three subscales) were analyzed with the Pearson Correlation Coefficient in order to test the relation between schizotypal traits and multisensory temporal acuity. It is worth noting that by taking the raw threshold values from VL trials and the absolute thresholds values from AL trials we can directly compare multisensory temporal acuity between the different experimental conditions (e.g. spatially congruent vs. incongruent; AL trials vs. VL trials). Moreover, doing so we have a common measure of temporal acuity for the correlational analyses. Thus, hereafter higher threshold values will always indicate that the $75 \%$ of perceived simultaneity reports are achieved at larger SOAs between cross-modal stimuli, indicating a reduced temporal acuity (i.e. a wider TBW).

\section{RESULTS}

Simultaneity Judgment Task 
As expected, the width of the time interval between the two stimuli influenced performance (Fig. 2, panel A). The ANOVA performed on simultaneity rate confirmed a significant main effect of SOA $\left(F_{(14,700)}=184.25, p<.001, \eta_{p}^{2}=0.787\right)$. In contrast, the ANOVA did not show a significant main effect of spatial congruency $\left(\mathrm{F}_{(1,50)}=2.22, \mathrm{p}=.142, \eta_{\mathrm{p}}^{2}=0.043\right)$ nor an interaction between spatial congruency and $\operatorname{SOA}\left(\mathrm{F}_{(14,700)}=1.140, \mathrm{p}=.318, \eta_{\mathrm{p}}^{2}=0.022\right)$.

When looking at the differences between thresholds as a function of the spatial congruency, we found a statistically significant threshold difference between the congruent and the incongruent spatial condition only in AL trials (one tail t-test: $t_{(50)}=-2.062, \mathrm{p}=.022, \mathrm{~d}=0.289$ ) (Fig. 2, panel B). Congruently, there was also a significant difference in the mean simultaneity rate in AL trials between the congruent and the incongruent spatial condition (one tail t-test: $t_{(50)}=1.772, p=.041, d=0.248$ ). Regarding the exploration of possible asymmetries as a function of the leading sense, the analysis showed that thresholds differed significantly between AL and VL trials both in the congruent $\left(\mathrm{t}_{(50)}=\right.$ $-6.120, \mathrm{p}<.001, \mathrm{~d}=0.857)$ and in the incongruent spatial condition $(\mathrm{t}(50)=-4.251, \mathrm{p}<.001, \mathrm{~d}=$ 0.595) (Fig. 2, panel B). Finally, we analyzed differences between AL and VL trials regardless of the spatial congruency (Fig. 3). The analyses showed statistically significant differences in the threshold $(\mathrm{t}(50)=-7.057, \mathrm{p}<.001, \mathrm{~d}=0.988)$ and in the simultaneity rate $(\mathrm{t}(50)=-9.879, \mathrm{p}<.001, \mathrm{~d}=1.383)$. Overall, the pattern of results is in line with a generally higher multisensory temporal precision when audition is the leading sense.

The results of our study could be compared with other results obtained from an SJ task in a classic laboratory setting. The study by Fenner and colleagues (2020), in particular, is suitable to this aim since is largely comparable to the present study in terms of SOAs employed and because the auditory presentation was made also in that case with headphones/earbuds. Differently from the present study, however, Fenner et al. did not compare spatial congruency vs. incongruency of AV stimuli and did not employ an AL condition, but tested only VL trials. In our study, the observed mean simultaneity rate at each SOAs in the VL condition (averaged between spatially congruent and incongruent trials) was as follows: $50 \mathrm{~ms}=0.93,100 \mathrm{~ms}=0.92,150 \mathrm{~ms}=0.82,200 \mathrm{~ms}=0.69,250 \mathrm{~ms}=0.54,300 \mathrm{~ms}$ 
$=0.37,350 \mathrm{~ms}=0.26$. Fenner and colleagues (2020) reported the following results (estimated from

the figure on p. 6): $50 \mathrm{~ms} \cong 0.87,100 \mathrm{~ms} \cong 0.82,150 \mathrm{~ms} \cong 0.78,200 \mathrm{~ms} \cong 0.70,250 \mathrm{~ms} \cong 0.64$,

$300 \mathrm{~ms} \cong 0.54,350 \mathrm{~ms} \cong 0.50$.

\section{[Insert Figure 2 about here]}

A.

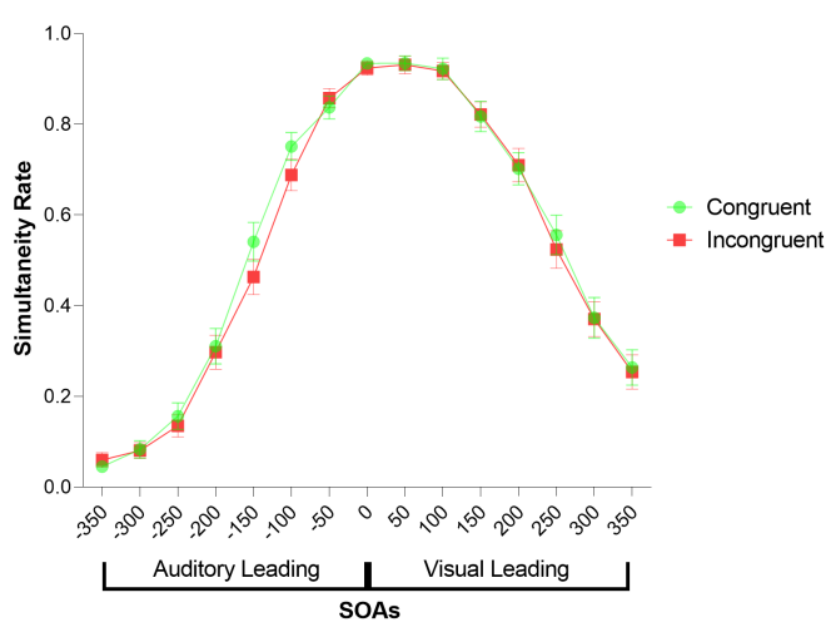

B.

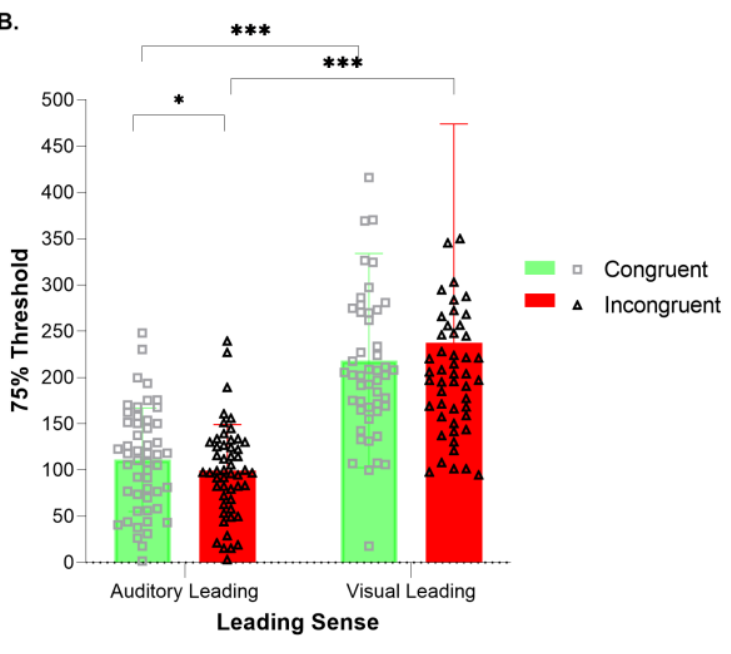

Figure 2. A) Repeated measures ANOVA performed on simultaneity rate revealed a significant main effect of SOA, revealing that the variability of the width of the time interval between the two stimuli influenced performance. This analysis did not show a significant main effect of the spatial congruency nor an interaction between spatial congruency and SOA. The error bars indicate the standard error of the mean (SEM). B) The figure shows the differences between $\mathrm{AL}$ and VL trials in the different conditions of spatial congruency. The error bars indicate the standard error of the mean $(\mathrm{SEM}) ; *$ indicates $\mathrm{p}<.05 ; * * *$ indicates $\mathrm{p}<.001$ as obtained from paired-sample t-tests.

\section{[Insert Figure 3 about here]}

A.

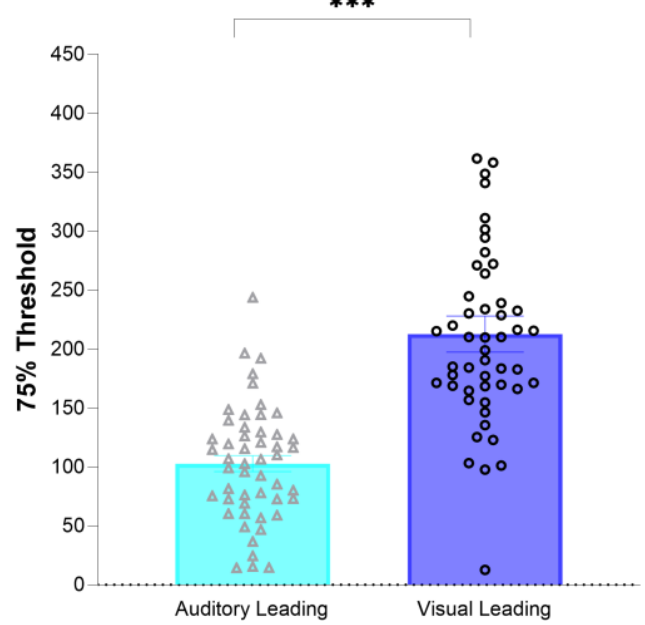

B.

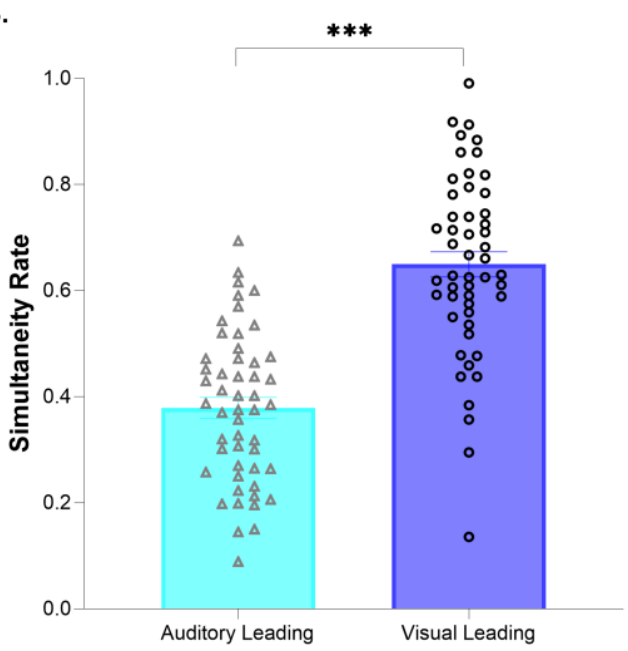

Figure 3. The figures highlight the significant asymmetries between AL and VL trials regardless of the spatial congruency of audiovisual stimuli. The analysis showed statistically significant differences both in the threshold and simultaneity rate (*** indicates $\mathrm{p}<.001$ as obtained from paired-sample t-tests). The error bars indicate the standard error of the mean (SEM) 


\section{Correlation between audio-visual temporal acuity and schizotypal traits}

The analysis of the Pearson correlation coefficient between measures derived from the spatial congruent condition of the SJ task and the SPQ scores (Fig. 4) showed: i) a significant positive correlation between the AL simultaneity rate and the "Disorganized" subscore of the SPQ $(r=.313$, $\mathrm{p}=.025)$; ii) a significant positive correlation between AL thresholds and the "Disorganized" subscore of the SPQ $(r=.417, \mathrm{p}=.002)$.

The analysis of the Pearson correlation coefficient between measures derived from the spatial incongruent condition of the SJ task and the SPQ scores (Fig. 4) showed: i) a significant positive correlation between the AL thresholds and the "Disorganized" subscore of the SPQ $(r=.335, \mathrm{p}=$ .016); ii) a significant positive correlation between the AL simultaneity rate and the "Disorganized" subscore of the SPQ $(r=.278, p=.049)$.

As predicted based on previous studies, the pattern of correlations is consistent with a lower multisensory temporal acuity, as indexed by the raw simultaneity rates and thresholds of the psychometric curve, in the presence of more schizotypal traits. However, it is worth noticing that this correlation was specific for AL trials, and no significant correlations emerged between schizotypal traits and VL thresholds or simultaneity rates.

[Insert Figure 4 about here] 


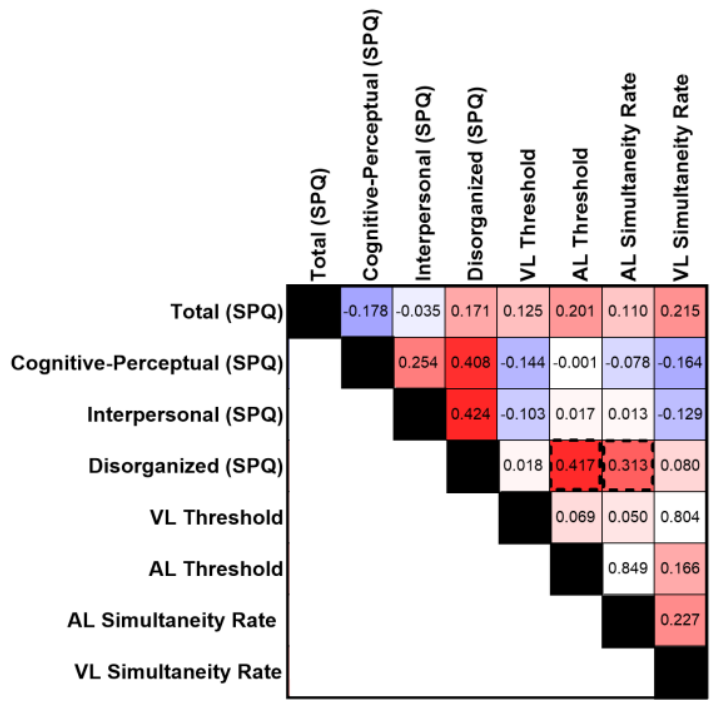

Congruent Condition

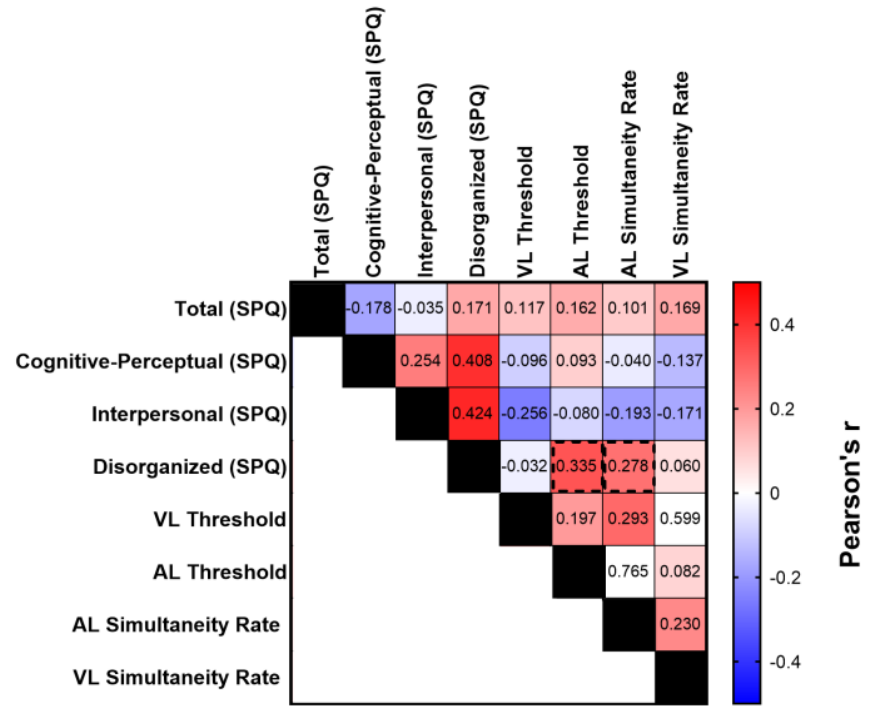

Incongruent Condition

Figure 4. The correlation matrix highlights the associations revealed by the Pearson correlation coefficient between the measures of the SJ task and schizotypal traits as measures by the SPQ. Statistically significant relationships between the measures examined are indicated as dashed black squares in the cells of the matrices. AL= auditory-leading trials; $\mathrm{VL}=$ visual-leading trials.

\section{DISCUSSION}

The current study represents a first empirical report on the possibility of adequately administering a SJ paradigm through a web-based platform. Our results confirmed that the response pattern of perceived simultaneity of audiovisual stimuli varies according to the time interval between the two stimuli, with simultaneity reports more common when the SOAs between the auditory and visual stimulus was brief, and a clear decline in perceived simultaneity at longer SOAs. This pattern of results is in line with classic lab-based studies available in the literature (e.g. Zampini et al., 2005; Vatakis et al. 2008; Stevenson et al., 2012; Stevenson et al., 2017), providing a proof-of-concept that the results obtained from the present web-based SJ task closely mimic those obtained in controlled laboratory contexts. Such a finding opens up a realm of potential new studies that target underrepresented participant populations (e.g., not just university students) and big data approaches with large sample sizes. 
While the influence of the critical role of the temporal relationship between incoming audiovisual stimuli on multisensory integration has been extensively studied (for a review, see: Zhou, Cheung \& Chan, 2020), less is known about how the leading sense influences multisensory temporal acuity. To further investigate this question, we separately analyzed the left side (AL) and the right side (VL) of the psychometric curve to assess whether the individual thresholds differed as a function of the leading sense, and whether this also interacts with the spatial congruency factor. The results indicate a lower threshold in AL trials, i.e. a rightward shift of the psychometric curve, when the presentation of the audiovisual stimuli was spatially congruent compared to the condition of spatial incongruency. This data supports previous studies showing that subjects restrict their temporal acuity, becoming better in discriminating and detecting asynchrony, when the stimuli appear in different spatial positions, although this same pattern was previously highlighted for both sides of the psychometric curve (both AL and VL trials; Zampini et al., 2005; Stevenson et al., 2012), and not just for AL trials as found here.

To better investigate the possible asymmetries in multisensory temporal processes as a function of the leading sense, we focused on the comparison between AL and VL conditions regardless of spatial congruency. The direct comparison between AL and VL trials that revealed significant differences in thresholds, reflecting increased temporal acuity when audition was the leading sense. The replication of this common pattern in a web-based platform is noteworthy, considering that the neurocomputational processes underlying cross-modal integration in AL and VL trials might be substantially different. Accordingly, recent evidence has shown that the distribution obtained from the simultaneity rate leads to a narrower size of the TBW in AL trials, and a broader size for VL trials, suggesting that such integrative processes are not symmetrical, and that temporal binding of audiovisual information is regulated by two distinct mechanisms of multisensory interactions that depend on the order of presentation of these stimuli (Conrey \& Pisoni, 2006; Keetels \& Vroomen, 2012; Wassenhove, 2013 Cecere, Gross, \& Thut, 2016). In support of these asymmetries highlighted at the behavioral level there are also studies showing that the leading sense leads to markedly different 
neural processes, identifiable in different EEG/ERP topographical patterns for AL and VL trials, in support of a dual-route model of audiovisual integration that would depend on the leading sense (Cecere et al., 2017).

As suggested by Cecere and colleagues (2016), this neurocognitive asymmetry between AL and VL in the temporal process of multisensory integration could be explained by the processes of a crossmodal phase reset in certain frequency bands of brain oscillations, in which the sampling mechanisms of one sensory modality interacts with the other modality (Lakatos et al., 2009; Romei et al., 2012; Mercier et al., 2013). Critically, for multisensory processes related to audiovisual stimuli the nature of cross-modal phase reset depends on the leading sense (Thorne \& Debener, 2014). Typically, in the AL condition, the phase reset process signals the pre-imminence of an input to the visual system, by means of an attentional low-level mechanism, capable of explaining the efficiency in multisensory integration processes. On the other hand, in the VL condition the phase reset process seems to be driven by higher-level prediction mechanisms, which results in a lower accuracy in the judgments of asynchrony perception (Thorne \& Debener, 2014). Hence, the asymmetry between the two leading conditions (AL and VL) in the temporal integration processes revealed by our results supports prior evidence suggesting that the processes underlying the two conditions are at least somewhat independent, highlighting better multisensory temporal acuity for the condition AL, compared to the VL. This result assumes considerable importance in the evaluation of multisensory temporal processes in clinical populations in which these processes are anomalous, favoring the specific identification of sensory/perceptual and cognitive aspects involved in these disorders.

It is interesting to note that the difference in threshold between congruent and the incongruent spatial condition was found only in AL trials. In the case of an auditory-leading trial, the sound was presented first and thus it is possible that the spatial location of that sound was represented more strongly compared to VL trials, in which the visual flash, given its superior spatial resolution, might have "captured" the sound. Given the noted strength of visual capture and the "ventriloquist effect", the 
spatial incongruency might have been resolved differently. Only in the AL condition would the spatial location of the auditory stimulus be processed and determined first, before capture occurs.

The results obtained here suggest the feasibility of performing a web-based assessment of multisensory temporal integration with a SJ task that has been typically confined to controlled laboratory settings. Considering that the need to expand the average sample size employed in behavioral studies is an essential factor for increasing the validity and reliability of evidence in the psychological science field, the present study brings an example of how use of online psychophysical testing might lead to significant benefits in this sense. While online experimentation has revealed enormous potential for studying large samples of participants in controlled and optimized research settings (Birnbaum, 2001; Sauter, Draschkow \& Mack, 2020), psychophysical studies online deserve special attention due to their specific nature. Due to the lack of control for the context surrounding an online environment, running psychophysical studies online is particularly challenging given that parameters such as the viewing distance and stimulus size, which are often critical factors in psychophysics experimentation, are not fully controlled. In this regard, new methods are being developed in order to obtain a controlled and reliable experimental context. In a recent study $\mathrm{Li}$ and colleagues (2020), for example, introduced a new method capable of estimating the participant's viewing distance by detecting the eccentricity of their blind spot location. This methodological approach is able to automatically adjust and control the geometric configuration of the stimuli based on the estimate of the individual's viewing distance of each participant in the experiment, overcoming the limits deriving from psychophysical experiments conducted online.

Another main purpose of the present study was to address the analysis of the possible link between a higher presence of schizotypal traits investigated with the SPQ, and a lower temporal multisensory acuity indexed by the web-based SJ task. The presence in the non-clinical population of traits belonging to SCZ stimulates the investigation of potential differences in multisensory temporal capacities - typically compromised in patients with SCZ - also in the general population with no known history of this condition. This possibility is suggested by recent evidence that has revealed a 
strong relationship between impairments in the temporal processes of multisensory integration and the presence of schizotypal traits in the general population (Ferri et al., 2018; Fenner at al., 2020). In particular, Dalal and colleagues (2021) recently highlighted that individuals with lower multisensory acuity have a higher level of schizotypal traits, especially in the cognitive-perceptual domain. Specifically, we examined the relationship between the different domains investigated by the SPQ and the different measures obtained in the audiovisual SJ task. Our findings, in agreement with previous laboratory-based studies, showed that lower multisensory acuity was generally associated with a higher presence of schizotypal traits. When we examined the relationship between the different schizotypal domains and multisensory integration as a function of the leading sense and spatial congruency, some specific patterns emerged. In conditions of spatial congruency, higher individual thresholds and mean simultaneity rates in AL trials (i.e. decreased temporal acuity) positively correlated with the score obtained in the "Disorganized" subscale of the SPQ. Also, in conditions of spatial incongruency results pointed in the same direction: higher thresholds and mean simultaneity rates in AL trials positively correlated with the score obtained in the "Disorganized" subscale of the SPQ. To summarize, our results are in line with the previous literature, supporting the evidence that a lower multisensory temporal acuity is correlated with the presence of more schizotypal traits (Overton, 2015; Ferri et al., 2018; Zhou et al., 2020; Dalal et al., 2021), which in turn agree with evidence obtained in individuals with a clinical diagnosis of SCZ (Haß et al., 2017; Stevenson et al., 2017; Noel, Stevenson \& Wallace, 2018; for a review, see Zhou et al., 2018). Furthermore, considering that we pointed out, in line with some previous studies, the existence of asymmetries in temporal multisensory processes associated with the leading sense, it would be interesting to further explore this aspect in SCZ and as a function of the schizotypal traits, and in other clinical populations with known anomalies in multisensory integration such as Autism Spectrum Disorders (Wallace \& Stevenson, 2015; Zhou et al., 2018). Our preliminary data suggest that disorganized behaviors correlated selectively with higher temporal imprecision in AL trials. 
Interestingly, the fact that a lower multisensory temporal acuity was selectively associated with an increase in the schizotypal behaviors in the cognitive-perceptual and disorganized domains connect to some etiological theories of schizophrenia that postulate multisensory integration anomalies as critical factors at the basis of the pathogenesis of the disorder. The "Panmodal processing imprecision hypothesis of schizophrenia" (Javitt et al., 1999; Bates, 2005) suggests that individuals with SCZ possess impairments in the cognitive-perceptual system, which translates into a less accurate processing of sensory information compared to neurotypical individuals. At the neural level, the "Disconnection hypothesis" (Friston et al., 2016) argues that an aberrant multisensory integration process in this clinical population is produced by an abnormal synaptic connection, and by a lower neural connectivity. Hence, a lower (or atypical) connectivity between the auditory and visual system, could explain the impairment present in the multisensory integration processes since reduced connectivity could lead to slower or less efficient MSI. Such multisensory integration deficits would then lead to a reduced temporal acuity and, in turn, to an inaccurate temporal processing of incoming sensory stimuli, which might play a role in sensory overload and to an ambiguous and imprecise perceptual experiences (Thakkar et al., 2011; Sartorato et al., 2017); both these characteristics are typically manifested by schizophrenic individuals (Stevenson \& Wallace, 2018, Haß et al., 2017; Zhou et al., 2018). The link between lower multisensory acuity and higher schizotypal traits in the cognitive-perceptual and disorganized domains suggest a close relationship between the etiology of SCZ and the ability to integrate inputs from different sensory modalities. More broadly, it suggests that the multisensory temporal integration deficit may be a core symptom and a core etiological factor of the disorder, to which more attention should be paid in the future. Indeed, MSI might be a useful marker for at-risk individuals that could be identified prior to the typical onset age of schizophrenia. Markers for schizophrenia may be of particular relevance given theories that emphasize the role of epigenetics and environmental stressors in influencing the likelihood that schizotypal traits (and the relevant genes) develop into full schizophrenia (for example: Mirsky and Duncan, 1986; van Os, Kenis \& Rutten, 2010). 
One aspect which would deserve additional investigation is for example whether temporal processing is anomalous only in the context of cross-modal integration or whether it could be seen also in unisensory domains (such as the differences in thresholds for segregating two visual flashes as separate percepts: Ronconi \& Melcher, 2017; Ronconi et al., 2017; 2018; 2020), an aspect which is still controversial in the literature (Stone et al., 2011; Stevenson et al., 2017; Zhou et al., 2018; Noel, Stevenson \& Wallace, 2018). In future work, it would also be valuable to see whether such impairments are amenable to training in order to intervene in clinical populations. There is increasing evidence that multisensory TBWs are at least somewhat plastic and can be narrowed through perceptual learning (Powers et al., 2009; Powers, Hevey \& Wallace, 2012; Stevenson et al., 2013; Powers, Hillock-Dunn \& Wallace, 2016; Cecere, Gross \& Thut, 2016, McGovern et al., 2016). Even training that is restricted to the visual modality has shown benefits in multisensory temporal processes (Stevenson et al., 2013), i.e. a narrowing of the multisensory TBW. A recent study also demonstrated effects of TBW training at the neural level: individuals who showed a narrower TBW after a perceptual learning training with audiovisual stimuli showed greater activity of the parietal and occipital beta frequencies (Theves et al., 2020).

In conclusion, the current findings provide further evidence for the possibility of performing accurate web-based psychophysical assessment using audio-visual multisensory paradigms that were previously limited to laboratory settings. The results obtained also provide additional evidence supporting the leading sense asymmetries in multisensory integration processes. Finally, our pattern of results provides strong evidence in support of previous studies that have highlighted temporal multisensory integration deficits as a hallmark of SCZ and its broader phenotype. Considering that this work represents a first attempt in this direction, further work may move beyond this proof-ofconcept finding and extend the investigation to other commonly used multisensory psychophysical tasks, in the hope of establishing the web-based option as a valid alternative to address complex aspects of human multisensory perception outside an experimental psychology laboratory and for a more representative demographic of participants. 


\section{REFERENCES}

- Andersen, T. S., Tiippana, K., \& Sams, M. (2004). Factors influencing audiovisual fission and fusion illusions. Cognitive Brain Research, 21(3), 301-308.

- Balz, J., Roa Romero, Y., Keil, J., Krebber, M., Niedeggen, M., Gallinat, J., \& Senkowski, D. (2016). Beta/gamma oscillations and event-related potentials indicate aberrant multisensory processing in schizophrenia. Frontiers in psychology, 7, 1896.

- Bates, T. C. (2005). The panmodal sensory imprecision hypothesis of schizophrenia: reduced auditory precision in schizotypy. Personality and individual differences, 38(2), 437-449.

- Berinsky, A. J., Huber, G. A., \& Lenz, G. S. (2012). Evaluating online labor markets for experimental research: Amazon. com's Mechanical Turk. Political analysis, 20(3), 351-368.

- Birnbaum, M. H. (2001). Introduction to behavioral research on the Internet.

- Bishop, C. W., \& Miller, L. M. (2009). A multisensory cortical network for understanding speech in noise. Journal of cognitive neuroscience, 21(9), 1790-1804.

- Buhrmester, M., Kwang, T., \& Gosling, S. D. (2016). Amazon's Mechanical Turk: A new source of inexpensive, yet high-quality data?

- Cecere, R., Gross, J., \& Thut, G. (2016). Behavioural evidence for separate mechanisms of audiovisual temporal binding as a function of leading sensory modality. European Journal of Neuroscience, 43(12), 1561-1568.

- Cecere, R., Gross, J., Willis, A., \& Thut, G. (2017). Being first matters: topographical representational similarity analysis of ERP signals reveals separate networks for audiovisual temporal binding depending on the leading sense. Journal of Neuroscience, 37(21), 52745287.

- Cohen, J., Collins, R., Darkes, J., \& Gwartney, D. (2007). A league of their own: demographics, motivations and patterns of use of 1,955 male adult non-medical anabolic 
steroid users in the United States. Journal of the International Society of Sports Nutrition, $4(1), 1-14$.

- Colonius, H., \& Diederich, A. (2004). Multisensory interaction in saccadic reaction time: a time-window-of-integration model. Journal of cognitive neuroscience, 16(6), 1000-1009.

- Conrey, B., \& Pisoni, D. B. (2006). Auditory-visual speech perception and synchrony detection for speech and nonspeech signals. The Journal of the Acoustical Society of America, 119(6), 4065-4073.

- Dalal, T. C., Muller, A. M., \& Stevenson, R. A. (2021). The relationship between multisensory temporal processing and schizotypal traits. Multisensory Research, 1(aop), 1-19.

- Diederich, A., \& Colonius, H. (2015). The time window of multisensory integration: relating reaction times and judgments of temporal order. Psychological review, 122(2), 232.

- Ettinger, U., Meyhöfer, I., Steffens, M., Wagner, M., \& Koutsouleris, N. (2014). Genetics, cognition, and neurobiology of schizotypal personality: a review of the overlap with schizophrenia. Frontiers in psychiatry, 5, 18.

- Fenner, B., Cooper, N., Romei, V., \& Hughes, G. (2020). Individual differences in sensory integration predict differences in time perception and individual levels of schizotypy. Consciousness and Cognition, 84, 102979.

- Ferri, F., Nikolova, Y. S., Perrucci, M. G., Costantini, M., Ferretti, A., Gatta, V., ... \& Northoff, G. (2017). A neural "tuning curve" for multisensory experience and cognitiveperceptual schizotypy. Schizophrenia bulletin, 43(4), 801-813.

- Ferri, F., Venskus, A., Fotia, F., Cooke, J., \& Romei, V. (2018). Higher proneness to multisensory illusions is driven by reduced temporal sensitivity in people with high schizotypal traits. Consciousness and cognition, 65, 263-270.

- Fossati, A., Raine, A., Carretta, I., Leonardi, B., \& Maffei, C. (2003). The three-factor model of schizotypal personality: Invariance across age and gender. Personality and individual differences, 35(5), 1007-1019. 
- Foss-Feig, J. H., Kwakye, L. D., Cascio, C. J., Burnette, C. P., Kadivar, H., Stone, W. L., \& Wallace, M. T. (2010). An extended multisensory temporal binding window in autism spectrum disorders. Experimental Brain Research, 203(2), 381-389.

- Francisco, A. A., Jesse, A., Groen, M. A., \& McQueen, J. M. (2017). A general audiovisual temporal processing deficit in adult readers with dyslexia. Journal of speech, language, and hearing research, 60(1), 144-158.

- Friston, K., Brown, H. R., Siemerkus, J., \& Stephan, K. E. (2016). The dysconnection hypothesis (2016). Schizophrenia research, 176(2-3), 83-94.

- Grant, K. W., \& Seitz, P. F. (2000). The use of visible speech cues for improving auditory detection of spoken sentences. The Journal of the Acoustical Society of America, 108(3), 1197-1208.

- Hairston, W. D., Burdette, J. H., Flowers, D. L., Wood, F. B., \& Wallace, M. T. (2005). Altered temporal profile of visual-auditory multisensory interactions in dyslexia. Experimental Brain Research, 166(3-4), 474-480.

- Haß, K., Sinke, C., Reese, T., Roy, M., Wiswede, D., Dillo, W., ... \& Szycik, G. R. (2017). Enlarged temporal integration window in schizophrenia indicated by the double-flash illusion. Cognitive neuropsychiatry, 22(2), 145-158.

- Hillock-Dunn, A. R., Powers, A. R., \& Wallace, M. T. (2011). Binding of sights and sounds: age-related changes in multisensory temporal processing. Neuropsychologia, 49(3), 461-467.

- Hillock-Dunn, A., \& Wallace, M. T. (2012). Developmental changes in the multisensory temporal binding window persist into adolescence. Developmental science, 15(5), 688-696.

- Javitt, D. C., Liederman, E., Cienfuegos, A., \& Shelley, A. M. (1999). Panmodal processing imprecision as a basis for dysfunction of transient memory storage systems in schizophrenia. Schizophrenia bulletin, 25(4), 763-775.

- Kaganovich, N. (2017). Sensitivity to audiovisual temporal asynchrony in children with a history of specific language impairment and their peers with typical development: A 
replication and follow-up study. Journal of Speech, Language, and Hearing Research, 60(8), 2259-2270.

- Keetels, M., \& Vroomen, J. (2012). In The Neural Bases of Multisensory Processes (eds Murray M. \& Wallace M.) 147-178.

- Kuling, I. A., Van Eijk, R. L. J., Juola, J. F., \& Kohlrausch, A. (2012). Effects of stimulus duration on audio-visual synchrony perception. Experimental brain research, 221(4), 403412.

- Li, Q., Joo, S. J., Yeatman, J. D., \& Reinecke, K. (2020). Controlling for participants' Viewing Distance in Large-Scale, psychophysical online experiments Using a Virtual chinrest. Scientific reports, 10(1), 1-11.

- Lovelace, C. T., Stein, B. E., \& Wallace, M. T. (2003). An irrelevant light enhances auditory detection in humans: a psychophysical analysis of multisensory integration in stimulus detection. Cognitive brain research, 17(2), 447-453.

- Murray, M. M., Lewkowicz, D. J., Amedi, A., \& Wallace, M. T. (2016). Multisensory processes: a balancing act across the lifespan. Trends in Neurosciences, 39(8), 567-579.

- Martin, B., Giersch, A., Huron, C., \& van Wassenhove, V. (2013). Temporal event structure and timing in schizophrenia: preserved binding in a longer "now". Neuropsychologia, 51(2), $358-371$.

- Mason, W., \& Suri, S. (2012). Conducting behavioral research on Amazon's Mechanical Turk. Behavior research methods, 44(1), 1-23.

- Matthews, W. J., Stewart, N., \& Wearden, J. H. (2011). Stimulus intensity and the perception of duration. Journal of Experimental Psychology: Human perception and performance, 37(1), 303.

- McGovern, D. P., Roudaia, E., Newell, F. N., \& Roach, N. W. (2016). Perceptual learning shapes multisensory causal inference via two distinct mechanisms. Scientific reports, 6(1), 111. 
- Mirsky AF, Duncan CC (1986). Etiology and expression of schizophrenia: Neurobiological and psychosocial factors. Annual Review of Psychology, 37, 291-321).

- Noel, J. P., Łukowska, M., Wallace, M., \& Serino, A. (2016). Multisensory simultaneity judgment and proximity to the body. Journal of Vision, 16(3), 21-21.

- Noel, J. P., Stevenson, R. A., \& Wallace, M. T. (2018). Atypical audiovisual temporal function in autism and schizophrenia: similar phenotype, different cause. European Journal of Neuroscience, 47(10), 1230-1241.

- Overton, D. J. (2015). Audiovisual integration deficits in schizotypal personality and implications for populations diagnosed with schizophrenia (Doctoral dissertation).

- Pasqualotto, A., Dumitru, M. L., \& Myachykov, A. (2016). Multisensory integration: Brain, body, and world. Frontiers in Psychology, 6, 2046.

- Postmes, L., Sno, H. N., Goedhart, S., Van Der Stel, J., Heering, H. D., \& De Haan, L. (2014). Schizophrenia as a self-disorder due to perceptual incoherence. Schizophrenia research, 152(1), 41-50.

- Powers, A. R., Hillock, A. R., \& Wallace, M. T. (2009). Perceptual training narrows the temporal window of multisensory binding. Journal of Neuroscience, 29(39), 12265-12274.

- Powers, A. R., Hevey, M. A., \& Wallace, M. T. (2012). Neural correlates of multisensory perceptual learning. Journal of Neuroscience, 32(18), 6263-6274.

- Powers III, A. R., Hillock-Dunn, A., \& Wallace, M. T. (2016). Generalization of multisensory perceptual learning. Scientific reports, 6(1), 1-9.

- Raine, A. (1991). The SPQ: a scale for the assessment of schizotypal personality based on DSM-III-R criteria. Schizophrenia bulletin, 17(4), 555-564.

- Reips, U. D. (2002). Standards for Internet-based experimenting. Experimental psychology, 49(4), 243. 
- Roach, N. W., Heron, J., Whitaker, D., \& McGraw, P. V. (2011). Asynchrony adaptation reveals neural population code for audio-visual timing. Proceedings of the Royal Society B: Biological Sciences, 278(1710), 1314-1322.

- Ronconi, L., \& Melcher, D. (2017). The role of oscillatory phase in determining the temporal organization of perception: evidence from sensory entrainment. Journal of Neuroscience, 37(44), 10636-10644.

- Ronconi, L., Oosterhof, N. N., Bonmassar, C., \& Melcher, D. (2017). Multiple oscillatory rhythms determine the temporal organization of perception. Proceedings of the National Academy of Sciences, 114(51), 13435-13440.

- Ronconi, L., Busch, N. A., \& Melcher, D. (2018). Alpha-band sensory entrainment alters the duration of temporal windows in visual perception. Scientific reports, 8(1), 1-10.

- Ronconi, L., Melcher, D., \& Franchin, L. (2020). Investigating the role of temporal processing in developmental dyslexia: Evidence for a specific deficit in rapid visual segmentation. Psychonomic Bulletin \& Review, 27, 724-734.

- Royal, D. W., Carriere, B. N., \& Wallace, M. T. (2009). Spatiotemporal architecture of cortical receptive fields and its impact on multisensory interactions. Experimental brain research, 198(2-3), 127-136.

- Sartorato, F., Przybylowski, L., \& Sarko, D. K. (2017). Improving therapeutic outcomes in autism spectrum disorders: Enhancing social communication and sensory processing through the use of interactive robots. Journal of psychiatric research, 90, 1-11.

- Sauter, M., Draschkow, D., \& Mack, W. (2020). Building, hosting and recruiting: A brief introduction to running behavioral experiments online. Brain sciences, 10(4), 251.

- Schall, S., Quigley, C., Onat, S., \& König, P. (2009). Visual stimulus locking of EEG is modulated by temporal congruency of auditory stimuli. Experimental brain research, 198(23), 137-151. 
- Senkowski, D., Talsma, D., Grigutsch, M., Herrmann, C. S., \& Woldorff, M. G. (2007). Good times for multisensory integration: effects of the precision of temporal synchrony as revealed by gamma-band oscillations. Neuropsychologia, 45(3), 561-571.

- Spence, C. (2007). Audiovisual multisensory integration. Acoustical science and technology, 28(2), 61-70.

- Stevenson, R. A., \& Wallace, M. T. (2013). Multisensory temporal integration: task and stimulus dependencies. Experimental brain research, 227(2), 249-261.

- Stevenson, R. A., Fister, J. K., Barnett, Z. P., Nidiffer, A. R., \& Wallace, M. T. (2012). Interactions between the spatial and temporal stimulus factors that influence multisensory integration in human performance. Experimental Brain Research, 219(1), 121-137.

- Stevenson, R. A., Park, S., Cochran, C., McIntosh, L. G., Noel, J. P., Barense, M. D., ... \& Wallace, M. T. (2017). The associations between multisensory temporal processing and symptoms of schizophrenia. Schizophrenia research, 179, 97-103.

- Stevenson, R. A., VanDerKlok, R. M., Pisoni, D. B., \& James, T. W. (2011). Discrete neural substrates underlie complementary audiovisual speech integration processes. Neuroimage, 55(3), 1339-1345.

- Stevenson, R. A., Wilson, M. M., Powers, A. R., \& Wallace, M. T. (2013). The effects of visual training on multisensory temporal processing. Experimental brain research, 225(4), 479-489.

- Stone, D. B., Urrea, L. J., Aine, C. J., Bustillo, J. R., Clark, V. P., \& Stephen, J. M. (2011). Unisensory processing and multisensory integration in schizophrenia: a high-density electrical mapping study. Neuropsychologia, 49(12), 3178-3187.

- Talsma, D., Senkowski, D., \& Woldorff, M. G. (2009). Intermodal attention affects the processing of the temporal alignment of audiovisual stimuli. Experimental brain research, 198(2-3), 313-328. 
- Thakkar, K. N., Nichols, H. S., McIntosh, L. G., \& Park, S. (2011). Disturbances in body ownership in schizophrenia: evidence from the rubber hand illusion and case study of a spontaneous out-of-body experience. PloS one, 6(10), e27089.

- Theves, S., Chan, J. S., Naumer, M. J., \& Kaiser, J. (2020). Improving audio-visual temporal perception through training enhances beta-band activity. Neuroimage, 206, 116312.

- Tseng, H. H., Bossong, M. G., Modinos, G., Chen, K. M., McGuire, P., \& Allen, P. (2015). A systematic review of multisensory cognitive-affective integration in schizophrenia. Neuroscience \& Biobehavioral Reviews, 55, 444-452.

- Van Atteveldt, N. M., Formisano, E., Blomert, L., \& Goebel, R. (2007). The effect of temporal asynchrony on the multisensory integration of letters and speech sounds. Cerebral Cortex, 17(4), 962-974.

- van Os, J., Kenis, G., \& Rutten, B.P.F. (2010). The environment and schizophrenia. Nature, 468, 203-212.

- Van Wassenhove, V., Grant, K. W., \& Poeppel, D. (2007). Temporal window of integration in auditory-visual speech perception. Neuropsychologia, 45(3), 598-607.

- Vatakis, A., \& Spence, C. (2007). Crossmodal binding: Evaluating the "unity assumption" using audiovisual speech stimuli. Perception \& psychophysics, 69(5), 744-756.

- Vatakis, A., Navarra, J., Soto-Faraco, S., \& Spence, C. (2008). Audiovisual temporal adaptation of speech: temporal order versus simultaneity judgments. Experimental brain research, 185(3), 521-529.

- Wallace, M. T., \& Stevenson, R. A. (2014). The construct of the multisensory temporal binding window and its dysregulation in developmental disabilities. Neuropsychologia, 64, 105-123.

- Wallace, M. T., Roberson, G. E., Hairston, W. D., Stein, B. E., Vaughan, J. W., \& Schirillo, J. A. (2004). Unifying multisensory signals across time and space. Experimental Brain Research, 158(2), 252-258. 
- Wilkinson, L. K., Meredith, M. A., \& Stein, B. E. (1996). The role of anterior ectosylvian cortex in cross-modality orientation and approach behavior. Experimental Brain Research, 112(1), 1-10.

- Woynaroski, T. G., Kwakye, L. D., Foss-Feig, J. H., Stevenson, R. A., Stone, W. L., \& Wallace, M. T. (2013). Multisensory speech perception in children with autism spectrum disorders. Journal of autism and developmental disorders, 43(12), 2891-2902.

- Zampini, M., Guest, S., Shore, D. I., \& Spence, C. (2005). Audio-visual simultaneity judgments. Perception \& psychophysics, 67(3), 531-544.

- Zhou, H. Y., Cheung, E. F., \& Chan, R. C. (2020). Audiovisual temporal integration: Cognitive processing, neural mechanisms, developmental trajectory and potential interventions. Neuropsychologia, 140, 107396.

- Zhou, H. Y., Cai, X. L., Weigl, M., Bang, P., Cheung, E. F., \& Chan, R. C. (2018). Multisensory temporal binding window in autism spectrum disorders and schizophrenia spectrum disorders: A systematic review and meta-analysis. Neuroscience \& Biobehavioral Reviews, 86, 66-76.

- Zmigrod, L., \& Zmigrod, S. (2016). On the temporal precision of thought: individual differences in the multisensory temporal binding window predict performance on verbal and nonverbal problem solving tasks. Multisensory research, 29(8), 679-701. 
Authorship: D.M. and L.R. designed the study. F.C. conducted the experiments. G.M., F.C. and L.R. analyzed the data. G.M. and L.R. prepared Figures. G.M., F.C., D.M. and L.R. wrote the paper. All authors reviewed the manuscript.

Conflict of interest statement: The authors declare no competing interests 